\title{
Evaluation of 2D Biomechanical Models of the Knee of One-Leg Stand for Total Knee Arthroplasty Planning
}

\author{
Malte Asseln ${ }^{1 *}$, Jörg Eschweiler ${ }^{1,2}$, Anika Kiliclar ${ }^{1}$, Ines Kutzner ${ }^{3}$, Georg \\ Bergmann $^{3}$ and Klaus Radermacher ${ }^{1}$ \\ ${ }^{1 *}$ Chair of Medical Engineering, Helmholtz Institute for Biomedical Engineering, RWTH Aachen \\ University, Aachen, 52074, Germany \\ ${ }^{2}$ Department of Orthopaedics, Aachen University Clinic, RWTH Aachen University, Aachen, \\ 52074, Germany \\ ${ }^{3}$ Julius Wolff Institute, Charité - Universitätsmedizin Berlin, Berlin, 13353, Germany \\ asseln@hia.rwth-aachen.de
}

\begin{abstract}
Biomechanical models of the knee have the potential to predict joint mechanics and improve the therapeutic outcome. In case of total knee arthroplasty (TKA) knowledge on post-operative acting joint forces and its consideration in pre-operative planning might increase implant longevity and patient satisfaction. The goal of this study was to evaluate 2D biomechanical models of the knee suitable for pre-operative TKA planning and to validate them based on in vivo measurements of 9 patients treated with instrumented knee implants.

An extensive literature research on 2D biomechanical models of the knee with the requirement that patient-specific model adaption can be performed with data available in the conventional clinical workflow revealed the models of Maquet and Minns. Both models require one-leg stand AP long-leg radiographs and they output the resultant tibiofemoral joint force. For model validation, data from the freely accessible OrthoLoad database (www.orthoload.com) containing forces acting in orthopaedic implants have been used.

Looking at the results, Minns's model was in the same range as the reference forces (deviation: $-55 \% \mathrm{BW}$ to $+80 \% \mathrm{BW}$ ) as well as Maquet's (deviation: $-80 \% \mathrm{BW}$ to +50 $\% \mathrm{BW}$ ). Overall, the root-mean-square error (RMSE) was smallest for Minns' model (48.36 \% BW against $51.48 \% \mathrm{BW}$ ). The patient-specific adaptation process plus simulation took 7 to 8 minutes making the models applicable in the conventional clinical workflow with acceptable costs.

In terms of clinical application, the models' output might be consulted, e.g. to minimize the magnitude of the resultant joint force, target a specific orientation or load
\end{abstract}


distribution in order to consider mechanical factors which might be associated with premature implant failure.

\section{Introduction}

Biomechanical models of the knee have the potential to predict joint mechanics and improve the therapeutic outcome. In case of total knee arthroplasty (TKA) knowledge on post-operative acting joint forces, e.g. magnitude and distribution, and its consideration in pre-operative planning might increase implant longevity and patient satisfaction.

Today, in the conventional clinical routine, TKA planning is based on the evaluation of 2D geometrical anterior-posterior (AP) and sagittal radiographs (Wirtz, 2011). Then, implant alignment is oriented towards the anatomical and mechanical leg axis, respectively. Thereby, biomechanical knowledge is only considered indirectly by assuming that neutral mechanical alignment establishes mechanical equilibrium of the medial and lateral compartment in the coronal plane and thus, minimizing shear forces of the prosthesis or theoretically maximizing longevity. However, Bellemans et al. questioned whether neutral mechanical alignment in TKA is normal for all patients after discovering that one fourth of the population had at least $3^{\circ}$ of natural varus alignment once they have reached skeletal maturity (Bellemans et al., 2012). Additionally, the results of Bonner et al. and Morgan et al. demonstrated that general neutral mechanical alignment is likely insufficient (Bonner et al., 2011; Morgan et al., 2008). Therefore, optimal patient-specific alignment targets still need to be defined.

The goal of this study was to evaluate 2D biomechanical models of the knee suitable for preoperative TKA planning and to validate them based on in vivo measurements of 9 patients treated with instrumented knee implants.

\section{Materials and Methods}

An extensive literature research on 2D biomechanical models of the knee with the requirement that patient-specific model adaption can be performed with data available in the conventional clinical workflow revealed the models of Maquet and Minns (Maquet and Pelzer, 1977; Minns, 1981). Both models require one-leg stand AP long-leg radiographs and they output the resultant tibiofemoral joint force (Figure 1). In case of Minns' model, also the medial/lateral distribution is provided. For model validation, data from the freely accessible OrthoLoad database (www.orthoload.com) containing forces acting in orthopaedic implants have been used. Here, for $n=9$ patients (mean age: 67.89 years, implanted side: 6 left and 3 right, mean weight: $92.41 \mathrm{~kg}$, mean height: $1.72 \mathrm{~m}$ ) in vivo joint force measurements and post-operative weight bearing AP long-leg radiographs were available. The models were adapted to the patients' situation by manually measuring landmarks in the patient-specific radiographs by a single trained observer based on a self-developed program in MATLAB. Meanwhile, the time was recorded for each measurement and the mean time was calculated for evaluation. Finally, model predictions were compared to their corresponding in vivo measurements published in the OrthoLoad database under "Standard Loads Knee Joint" (*_Stance.xlsx). The knee joint forces were expressed in \%BW, i.e. in percent of the patient's body weight $(\mathrm{BW})$, to allow a standardized comparison. 


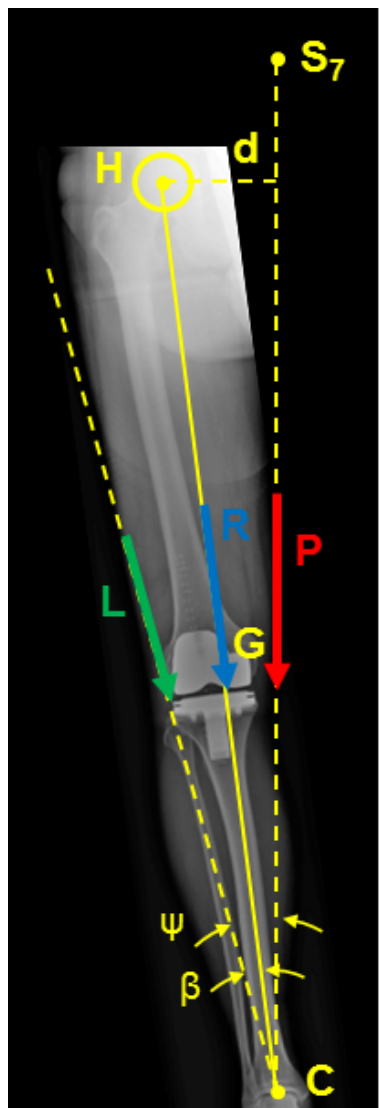

\begin{tabular}{|c|c|}
\hline Variable & Explanation \\
\hline $\mathrm{P}$ & Partial body weight $\left(0.929^{*}\right.$ BW) \\
\hline $\mathrm{L}$ & Lateral muscle force \\
\hline $\mathrm{R}$ & Resultant knee joint force \\
\hline $\mathrm{H}$ & Hip joint center \\
\hline G & Knee joint center \\
\hline $\mathrm{C}$ & Ankle joint center \\
\hline d & $\begin{array}{l}\text { Distance hip joint center }(\mathrm{H}) \text { to the } \\
\text { vertical }\end{array}$ \\
\hline $\mathrm{S}_{7}$ & $\begin{array}{l}\text { Center of mass of the partial body } \\
\text { weight }(P)\end{array}$ \\
\hline$\psi$ & $\begin{array}{l}\text { Angle between lateral muscle force } \\
\text { (L) and vertical }\end{array}$ \\
\hline$\beta$ & $\begin{array}{l}\text { Angle between lateral muscle force } \\
\text { (L) and mechanical axis of the tibia }\end{array}$ \\
\hline
\end{tabular}

Figure 1: Maquet's model with required parameters for patient-specific model adapation. Output is the resultant knee joint force

\section{Results}

The time for initialization was in the range of 2 minutes, landmark measurement in the range of 3 to 4 minutes and model application in the range of 2 minutes, giving a total duration of 7 to 8 minutes. Looking at the resultant joint forces large fluctuations were observed (Figure 2). However, Minns's model was in the same range as the reference forces (deviation: $-55 \% \mathrm{BW}$ to $+80 \% \mathrm{BW}$ ) as well as Maquet's (deviation: $-80 \% \mathrm{BW}$ to $+50 \% \mathrm{BW}$ ). Overall, the root-mean-square error (RMSE) was smallest for Minns' model (48.36 \%BW against 51.48\%BW). 


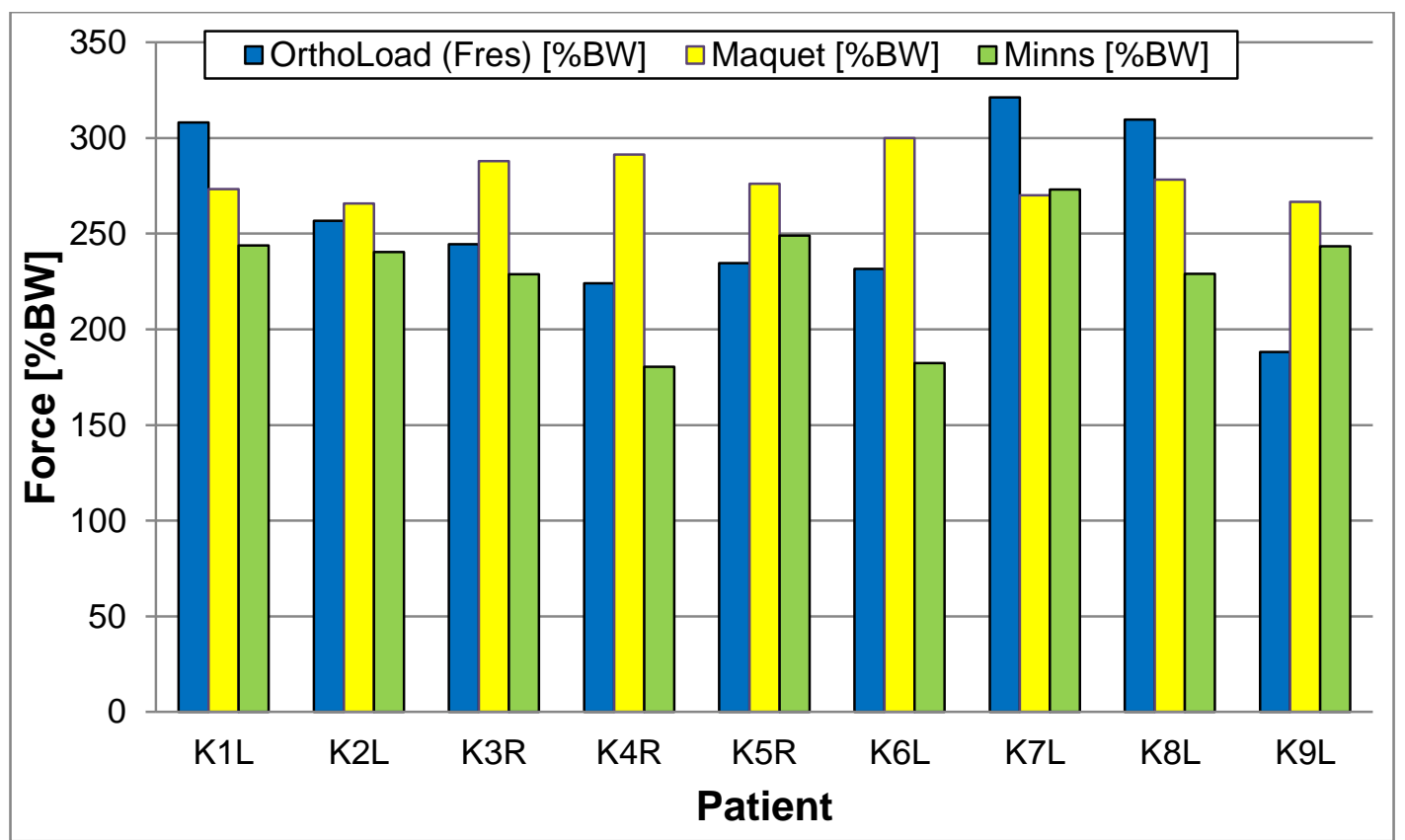

Figure 2: Resultant knee joint forces from Maquet's and Minns' model with corresponding in vivo force measurements

\section{Discussion}

In this study, known 2D biomechanical models capable to predict the resultant knee joint force have been individualized based on standard long leg radiographs, evaluated and validated by a comparison to in vivo forces measurements of overall 9 patients. This might be considered in TKA planning to obtain an optimal patient-specific implant alignment.

The patient-specific adaptation process took 7 to 8 minutes making the models applicable in the conventional clinical workflow with acceptable costs. Altogether, Minns' model showed the lowest deviations compared to the in vivo measurements. Although the differences in RMSE to Maquet's model were marginal, Minns' model is more complex offering principally the opportunity to evaluate the medial/lateral distribution. Compared to literature, the results were in the same range as presented by authors using sophisticated 3D modelling approaches. Stylianou et al. reported force differences to in vivo measurements of $44.9 \% \mathrm{BW}$ during squatting for a single patient (Stylianou et al., 2013). Chen et al. found deviations of $44.7 \% \mathrm{BW}$ during an entire gait cycle (Chen et al., 2014).

In terms of clinical application it has been demonstrated, that neutral mechanical alignment does not necessarily represent the patient-specific optimum. Here, the models' output might be consulted, e.g. to minimize the magnitude of the resultant joint force, target a specific orientation or load distribution in order to consider mechanical factors which might be associated with premature implant failure.

In conclusion, biomechanical models of the knee have the potential to improve TKA planning by providing additional information of the patient-specific biomechanical situation to the surgeon. Future work focuses on sensitivity studies, detailed distribution analysis, and optimization targets. 


\section{References}

Bellemans, J., Colyn, W., Vandenneucker, H., Victor, J., 2012. The Chitranjan Ranawat award: is neutral mechanical alignment normal for all patients? The concept of constitutional varus. Clinical orthopaedics and related research 470, 45-53.

Bonner, T.J., Eardley, W.G.P., Patterson, P., Gregg, P.J., 2011. The effect of post-operative mechanical axis alignment on the survival of primary total knee replacements after a follow-up of 15 years. The Journal of bone and joint surgery. British volume 93, 1217-1222.

Chen, Z., Zhang, X., Ardestani, M.M., Wang, L., Liu, Y., Lian, Q., He, J., Li, D., Jin, Z., 2014. Prediction of in vivo joint mechanics of an artificial knee implant using rigid multi-body dynamics with elastic contacts. Proceedings of the Institution of Mechanical Engineers. Part H, Journal of engineering in medicine 228, 564-575.

Maquet, P.G., Pelzer, G.A., 1977. Evolution of the maximum stress in osteo-arthritis of the knee. Journal of biomechanics 10, 107-117.

Minns, R.J., 1981. Forces at the knee joint: anatomical considerations. Journal of biomechanics $14,633-643$.

Morgan, S.S., Bonshahi, A., Pradhan, N., Gregory, A., Gambhir, A., Porter, M.L., 2008. The influence of postoperative coronal alignment on revision surgery in total knee arthroplasty. International orthopaedics 32, 639-642.

Stylianou, A.P., Guess, T.M., Kia, M., 2013. Multibody muscle driven model of an instrumented prosthetic knee during squat and toe rise motions. Journal of biomechanical engineering 135, 41008.

Wirtz, D.C., 2011. AE-Manual der Endoprothetik. Knie. Arbeitsgemeinschaft Endoprothetik, Berlin, Heidelberg. 